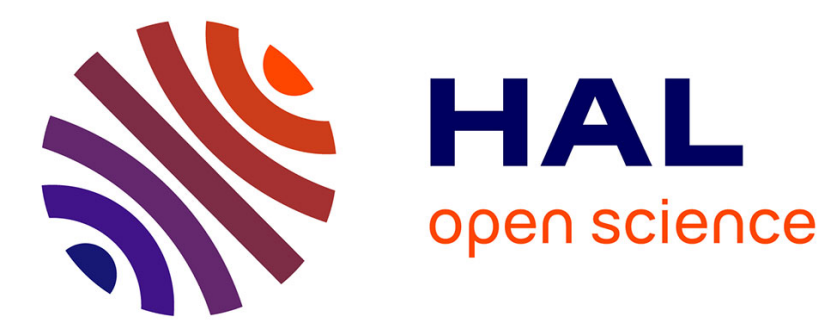

\title{
Elastic properties of GaS under high pressure by Brillouin scattering
}

\author{
Alain Polian, M. Besson, M. Grimsditch, H. Vogt
}

\section{To cite this version:}

Alain Polian, M. Besson, M. Grimsditch, H. Vogt. Elastic properties of GaS under high pressure by Brillouin scattering. Physical Review B: Condensed Matter (1978-1997), 1982, 25 (4), pp.2767 - 2775. 10.1103/PhysRevB.25.2767 . hal-01820712

\section{HAL Id: hal-01820712 https://hal.science/hal-01820712}

Submitted on 22 Jun 2018

HAL is a multi-disciplinary open access archive for the deposit and dissemination of scientific research documents, whether they are published or not. The documents may come from teaching and research institutions in France or abroad, or from public or private research centers.
L'archive ouverte pluridisciplinaire HAL, est destinée au dépôt et à la diffusion de documents scientifiques de niveau recherche, publiés ou non, émanant des établissements d'enseignement et de recherche français ou étrangers, des laboratoires publics ou privés. 


\title{
Elastic properties of GaS under high pressure by Brillouin scattering
}

\author{
A. Polian and J. M. Besson \\ Laboratoire de Physique des Solides - Associé au CNRS, \\ Université Pierre et Marie Curie, 4, place Jussieu, F-75230 Paris Cedex 05, France \\ M. Grimsditch* and H. Vogt \\ Max-Planck-Institut für Festkörperforschung, Heisenbergstrasse 7000 Stuttgart 80, \\ Federal Republic of Germany \\ (Received 14 April 1981; revised manuscript received 6 October 1981)
}

\begin{abstract}
Brillouin scattering from LA phonons in the layered semiconductor GaS has been measured in a diamond anvil cell up to $17.5 \mathrm{GPa}$. In the same range, linear compressibilities parallel and perpendicular to the $c$ axis have been determined, as well as the ordinary and extraordinary refractive indices. From these data the variations of the elastic constants $C_{11}$ and $C_{33}$ and the sound velocities under pressure are deduced. Brillouin data at ambient pressure give the five independent elastic constants of $\mathrm{GaS}\left(D_{6 h}\right)$, and the compressibilities computed from this set of $C_{i j}$ are found to coincide with the values observed in the high-pressure measurements. The optical-index anisotropy decreases to negligible values above $14 \mathrm{GPa}$. Comparison with an anisotropic modification of Murnaghan's equation of state shows that under high pressure, inter- and intra-layer forces still retain their individual behavior, although the former have become comparable in magnitude with the latter. Brillouin scattering in the ethanol-methanol mixture has been measured up to $17.5 \mathrm{GPa}$ and the variation of the elastic constant of the fluid is compared with Tait's and Murnaghan's equations of state.
\end{abstract}

\section{INTRODUCTION}

Brillouin scattering experiments have attractive aspects for high-pressure studies of elastic properties of matter. Ultrasonic measurements, which give the same type of information, have been attempted but, owing to experimental difficulties, comparatively few results have been obtained ${ }^{1-4}$ especially in the range of pressure above $5 \mathrm{GPa}$. Brillouin scattering, on the other hand, appears to be a practical method in this pressure range since it is well suited for use with diamond anvil cells because of the optical access. Hence, it can fully exploit the advantages of this recently developed technique: wide range of pressure $(100 \mathrm{GPa}$ and over $^{5}$ ), accurate pressure determination by the ruby luminescence scale, ${ }^{6,7}$ high homogeneity of the stress conditions, ${ }^{8}$ and no stringent requirements on the geometry of the sample, less than a microgram of which is needed as a rule. However, only two experiments have been reported to date, on $\mathrm{NaCl}$ up to 3,5 GPa (Ref. 9) and very recently on solid hydrogen up to $20 \mathrm{GPa}^{10}$

In the present paper we report on new experimental Brillouin scattering results obtained for
$\mathrm{GaS}$ with a diamond anvil cell over the pressure range 0 to $19 \mathrm{GPa}$. Experiments were limited here only by the occurrence of a phase transition at 19 GPa. ${ }^{11}$ In addition to the Brillouin measurements the refractive indices and linear compressibilities were also determined.

Experiments are described in Sec. II. In Sec. III, the variation of elastic constants of GaS under pressure is discussed. Finally, in Sec. IV, the elastic behavior of the ethanol-methanol mixture used as a compression "fluid" is reported and shown to fit with classical equations of state.

\section{EXPERIMENTAL PART}

\section{A. Samples and pressurization method}

Gallium sulfide samples were grown by vapor transport. They were hexagonal needles with natural faces parallel to the $c$ axis and could be readily cleaved perpendicular to the $c$ axis. Diameters of the needles varied between 5 and $200 \mu \mathrm{m}$. In the first set of experiments plane-parallel slabs about $15 \mu \mathrm{m}$ in thickness and $150 \mu \mathrm{m}$ in diameter 
with the $c$ axis parallel to the incident radiation were used. In another geometry, needles about 15 $\mu \mathrm{m}$ in diameter and $150 \mu \mathrm{m}$ along the $c$ axis were used (see Fig. 1).

Our optical diamond cell was built after a design by S. Block and G. Piermarini. ${ }^{6}$ The effective aperture being only $15^{\circ}$, only geometries close to backscattering could be used. The Inconel 750 gasket was imprinted by the anvil faces, then a $300-\mu \mathrm{m}$ hole centered on the impression was bored and finally the hole was squeezed to $200-\mu \mathrm{m}$ diameter. The final thicknesss was in the range of 25 to 35 $\mu \mathrm{m}$. With the 4:1 methanol-ethanol mixture as a pressure-transmitting medium, we maintained homogeneous stress conditions at all pressures by rapidly increasing the strain up to the highest pressure of a given run, and then going down slowly by small pressure decrement along the procedure we have described previously. ${ }^{8,11}$ Pressure inhomogeneity was gauged by the overall broadening of the ruby emission peaks and was found to be less than $0.5 \mathrm{GPa}$ at $20 \mathrm{GPa}$.

\section{B. Compressibility measurements}

Compressibility determinations were made directly $^{12}$ by measuring the change in the sample dimension along the $c$ and $a$ directions, from enlarged microphotographs obtained under identical magnification. In Fig. 1, the $c$ axis of the sample

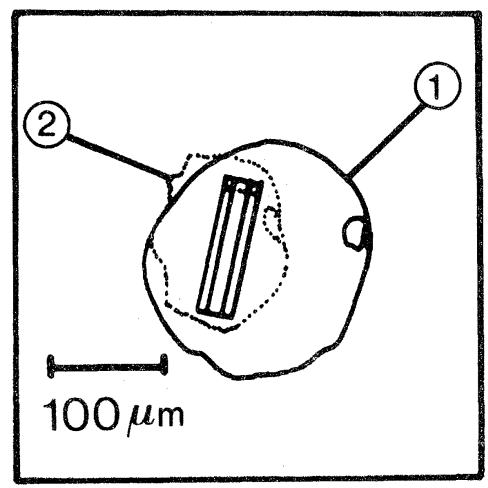

FIG. 1. Sketch of a GaS hexagonal needle in the diamond anvil cell. Prism faces are parallel to the $c$ axis and rest in the diamond plane. (1) is the outline of the Inconel gasket edges at ambient pressure. Dotted contour (2): same at $18.7 \mathrm{GPa}$. Shrinking of the crystal along the $c$ axis under pressure is shown by the dashed line at the top of the sample. A ruby chip is at the two o'clock position at both pressures. is in the plane of the cell and coincides with the length of the needle (about $150 \mu \mathrm{m}$ at ambient pressure). With this sample the decrease in length along the $c$ axis is directly measurable. The decrease along the $a$ axis was obtained with a $c$ cleavage plate (hexagonal slab) set parallel to the diamond faces.

Variations of $\Delta a / a_{0}$ and $\Delta c / c_{0}$ against pressure are shown in Fig. 2 up to the onset of the phase transition. ${ }^{11}$ The $c$ and $a$ axes vary quite differently under pressure. The high initial slope of $\Delta c / c_{0}$ reflects the weakness of interlayer forces and its strong curvature is in agreement with the highpressure behavior of Raman-active interlayer shear modes. ${ }^{11}$ On the other hand, $\Delta a / a_{0}$ versus pressure has nearly constant slope, in accordance with its being related to strong first-order intralayer bonds. We observe a continuous transition between a situation where $-\left(1 / a_{0}\right)(d a / d P)$

$<-\left(1 / c_{0}\right)(d c / d P)$, at low pressure, to the reverse situation at higher pressures. The low-pressure behavior is characteristic of two-dimensional networks but at high pressure the relative strength of the bonds is inverted, as indicated by the higher value of the in-plane compressibility. The important point here is that $\Delta a / a_{0}$ and $\Delta c / c_{0}$ do not become parallel at high pressure. This point will be utilized later in the discussion.

The compressibility values obtained from direct length measurements have been checked by comparing them with the compressibilities evaluated from the elastic constants measured at one atmosphere. Both methods give the same zero-pressure values within the experimental uncertainty, which

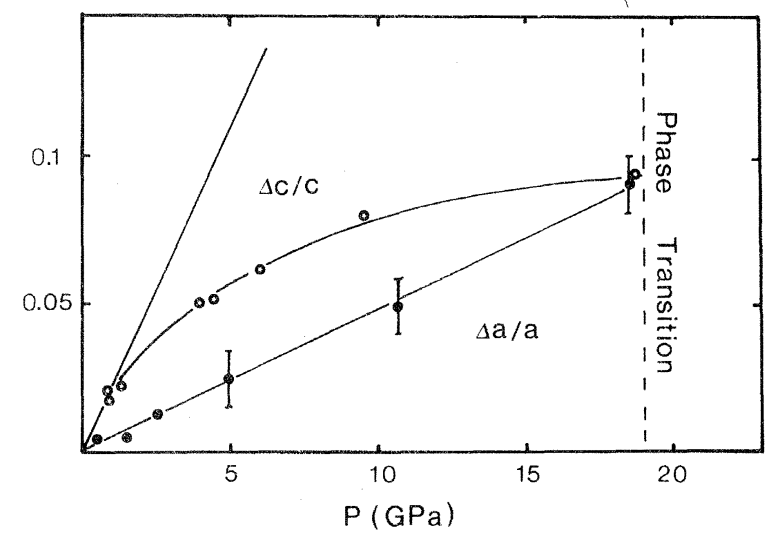

FIG. 2. Relative linear compression of GaS vs pressure. Empty stars: along the $c$ axis. Dots: along the $a$ direction (normal to the $c$ axis). Straight lines are computed from room pressure elastic constants [Eqs. (3) and (4)]. 
is about $15 \%$ on $\left[\left(1 / a_{0}\right)(d a / d P)\right]_{P=0}$ and less than $5 \%$ on $\left[\left(1 / c_{0}\right)(d c / d P)\right]_{P=0}$. This is a rough method but has the advantage of being straightforward and faster than high-pressure x-ray methods.

\section{Measurements of refractive indices}

The ordinary $n_{o}$ and extraordinary $n_{e}$ refractive indices of $\mathrm{GaS}$ at one atmosphere have been reported before. ${ }^{13}$ The one atmosphere value of $n_{o}$ is 2.63 at the $647.1-\mathrm{nm}$ wavelength of the krypton laser used in the present work. The ordinary refractive index as a function of pressure was directly measured by observing the interference fringe spectrum of plane parallel slabs with the faces perpendicular to the $c$ axis. The sample in the diamond cell at the focus of microscope was illuminated with white light which was analyzed in a T 800 Coderg triple grating monochromator. Transmission maxima are at

$$
2 n_{o} e \sigma=k \text {. }
$$

$e$ is the thickness of the sample at ambient pressure, $\sigma$ is the wave number of light, and $k$ is an integer (interference order) which can be calculated at ambient pressure.

Our thickness measurements (Fig. 2) combined with the fact that in this experiment, $k$ was small enough, enabled the same order to be identified and followed under pressure. Solving Eq. (1) at each pressure yields

$$
\left(n_{o}+\Delta n_{o}\right)(e-\Delta e) \sigma(P)=n_{o} e \sigma(0),
$$

from which we obtain the ordinary refractive index $n_{o}(P)$ at $647.1 \mathrm{~nm}$ given in Fig. 3. The extraordinary refractive index $n_{e}$ could, in principle, be

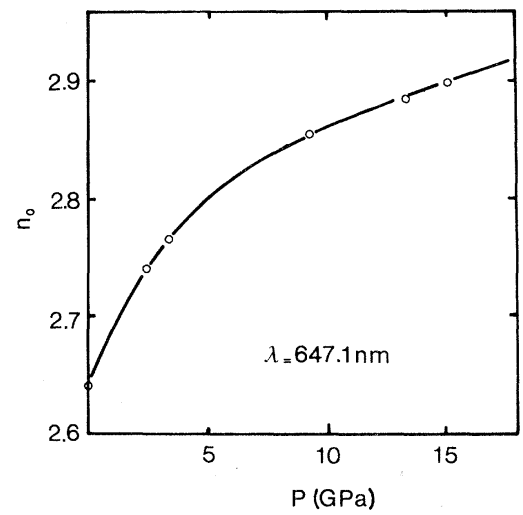

FIG. 3. Ordinary refractive index $n_{o}$ of GaS vs pressure at $647.1 \mathrm{~nm}$. determined by the same method but the small depolarization effect arising from the stresses in the diamonds would make the analysis difficult. However, we have been able to extract the pressure variation of $n_{e}$ from the Brillouin data.

\section{Brillouin scattering}

The instrument used in our experiments was a five-pass Fabry-Pérot interferometer. The high Brillouin efficiency of GaS allowed measurements without multiscanning and data accumulation even in the diamond cell. The 647.1-nm line of a $\mathrm{Kr}^{+}$ laser was used because of transparency conditions of $\mathrm{GaS}$ under high pressure ${ }^{11,14}$ at about $100-\mathrm{mW}$ incident power.

\section{Ambient pressure measurements}

Our measurements were performed in a variety of scattering geometries ranging from near forward to backscattering configurations in order to obtain a complete set of independent elastic constants. ${ }^{15}$

GaS belongs to the $D_{6 h}^{4}$ space group ${ }^{16}$ and has five independent elastic constants which are given in Table I. The values of the elastic constants were confirmed by direct ultrasonic measurements. The discepancies between our results and previous measurements ${ }^{17,18}$ will be discussed elsewhere. ${ }^{19}$ The compressibilities along $\left(\chi_{\|}\right)$and perpendicular $\chi_{\perp}$ to the $c$ axis can be expressed in terms of the elastic constants, yielding:

$$
\begin{aligned}
& \chi_{\perp}=-\frac{1}{a_{0}} \frac{\Delta a}{\Delta P}=\frac{C_{33}-C_{13}}{C_{33}\left(C_{11}+C_{12}\right)-2 C_{13}^{2}}, \\
& \chi_{\|}=-\frac{1}{c_{0}} \frac{\Delta c}{\Delta P}=\frac{C_{11}+C_{12}-2 C_{13}}{C_{33}\left(C_{11}+C_{12}\right)-2 C_{13}^{2}} .
\end{aligned}
$$

Using the elastic constants from Table I, we obtain $\chi_{\perp}=(5.1 \pm 0.1) \times 10^{-3} \mathrm{GPa}^{-1}$ and $\chi_{\|}=(23.9$ $\pm 0.4) \times 10^{-3} \mathrm{GPa}^{-1}$. These values represented by the straight lines in Fig. 2 are in excellent agreement with our compressibility measurements.

TABLE I. Elastic constants of GaS at room pressure and temperature in GPa.

\begin{tabular}{lcccc}
\hline \hline$C_{11}$ & $C_{33}$ & $C_{44}$ & $C_{12}$ & $C_{13}$ \\
\hline 122 & 38 & 9.8 & 33.4 & 8.9 \\
\hline
\end{tabular}




\section{High-pressure data}

Experiments performed in the pressure cell were restricted to backscattering geometries. This geometry, together with the two possible orientations of the crystal inside the cell enabled us to study phonons propagating parallel and perpendicular to the optic axis of the crystal. Furthermore, because only longitudinal modes are Brillouin active in this configuration, we obtain information only on $C_{11}$ and $C_{33}$ as a function of pressure.

In the experiments in the diamond cell, the Fabry-Pérot free spectral range was chosen so as to be an integral fraction of the frequency shift of the diamond Brillouin line. Thus, the diamond lines were "hidden" in the laser peak. Figure 4 depicts a typical spectrum recorded under those conditions. Brillouin peaks observed here are the LA mode of $\mathrm{GaS}$ and that of the pressure-transmitting medium (ethanol-methanol). The latter, which is a by-product of this experiment, will be discussed in Sec. IV. A rather surprising fact was that the incident laser beam, propagating along the axis of

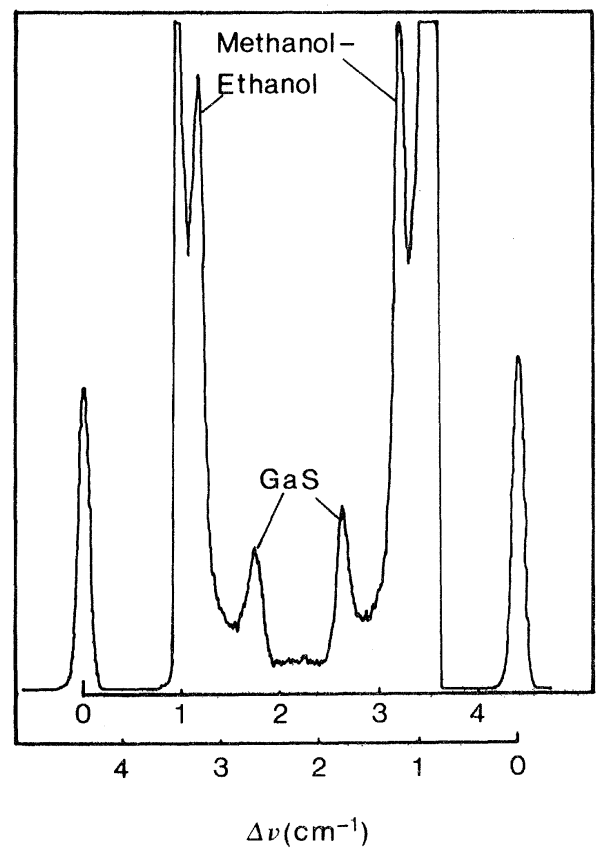

FIG. 4. Experimental recording of Brillouin spectrum showing lines of $\mathrm{GaS}$ and methanol-ethanol. The free spectral range is $4.383 \mathrm{~cm}^{-1}$ adjusted to locate the diamond peak at the laser wave number, the pressure is 9.6 $\mathrm{GPa}$, and the phonon is propagating perpendicular to the $c$ axis. Upper and lower abscissas apply to features left and right of the center, respectively. Configuration was $\overrightarrow{\mathrm{k}} \perp c \perp \overrightarrow{\mathrm{E}}$. both diamond windows, suffered comparatively little depolarization under stress. This enabled us to polarize the incident beam along a chosen direction. In particular, when scattering was observed from a face containing the optic axis of the crystal, the polarization of the incident light could be chosen either parallel or perpendicular to this axis. Brillouin lines associated with the ordinary and extraordinary refractive indices could be identified and thus provided a measure of the extraordinary component of the refractive index. Figure 5 shows the frequency shifts under pressure of the compression modes propagating parallel and perpendicular to the $c$ axis. Measurements were made with light polarized along [010] and [001] for $\vec{q} \|[100]$ and arbitrarily polarized light for $\overrightarrow{\mathrm{q}} \|[001]$.

\section{ANALYSIS OF RESULTS AND DISCUSSION}

\section{A. Compressibilities}

As already noted in Sec. II, compressibilities calculated from Eqs. 3 and 4 do fit with direct micrometric measurements and from these values we obtain the bulk compressibility $\chi=\chi_{\|}+2 \chi_{\perp}$ $=(34.1 \pm 0.6) \times 10^{-3} \mathrm{GPa}^{-1}$.

The most important result which can be seen in Fig. 2 is the evolution of the slopes of $\chi_{\|}$and $\chi_{\perp}$ versus pressure. At low pressure, the crystal is much more compressible along the $c$ axis than perpendicular to it, whereas the reverse situation occurs at high pressure. This fact clearly indicates

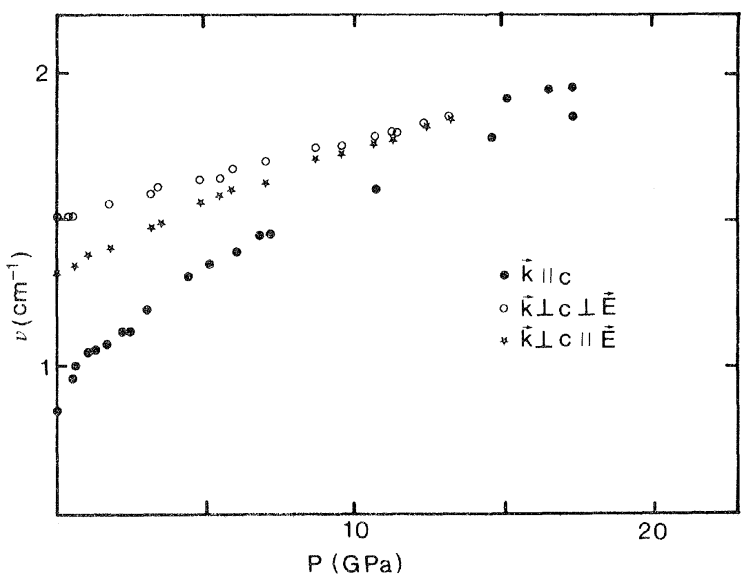

FIG. 5. Brillouin shift vs pressure for three backscattering geometries. Full circles for $\overrightarrow{\mathrm{k}} \| c$. Empty circles and stars refer to $\vec{k} \perp c$ and for ordinary and extraordinary refractive indices, respectively. 
that neither at low nor at high pressure are the inter- and intralayer bonds of the same nature. If it were the case, the compressibility curves would tend to be parallel. The fact that the slope of $\chi_{\|}$ becomes smaller than that of $\chi_{\perp}$ is consistent with the van der Waals type of bonding where the $r^{-12}$ term becomes dominant for small $r$, i.e. at high pressure.

\section{B. Optical anisotropy}

The upper two curves in Fig. 5, as mentioned above, represent the behavior of phonons propagating along the same direction. For $\overrightarrow{\mathrm{E}}$ (electric vector of the incident light) perpendicular to $c$, we use the ordinary index $n_{o}$ to compute the velocity, and for $\overrightarrow{\mathrm{E}}|| c$, the extraordinary index $n_{e}$. The ratio of ordinates thus gives the ratio $n_{e} / n_{o}$ versus $P$.

Since $n_{o}$ as a function of pressure is known (Sec. II), we can plot $n_{o}-n_{e}$ as a measure of the optical anisotropy (Fig. 6) at the wavelength $\lambda=647.1 \mathrm{~nm}$ of the laser. The index anisotropy decreases with pressure and becomes less than our experimental error at pressures of $14 \mathrm{GPa}$ and above. This is consistent with our work on Raman scattering and optical properties of GaS. ${ }^{11}$ We had then proposed that, at pressures in the range of 15 to $19 \mathrm{GPa}$, $\mathrm{GaS}$ did behave as though intra- and interlayer forces were comparable in magnitude before any phase transition occurred.

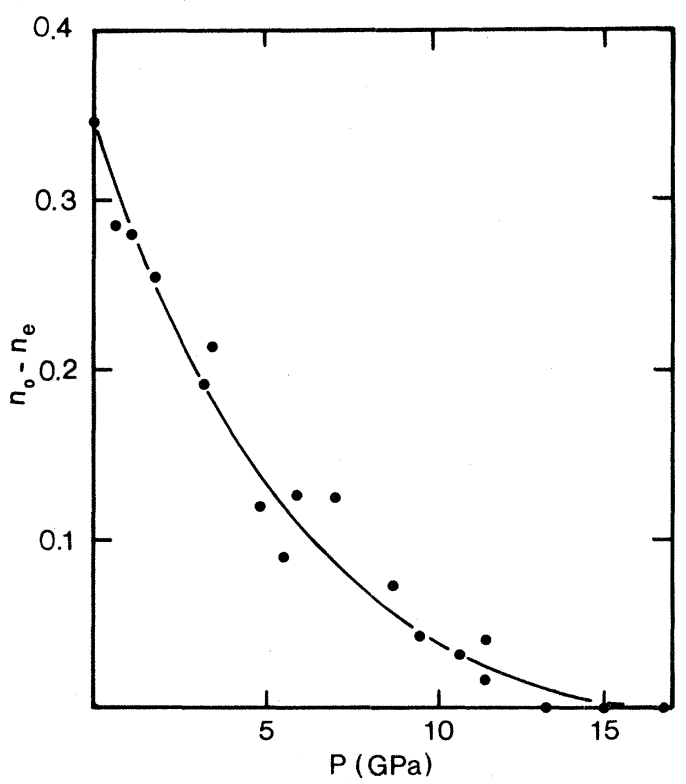

FIG. 6. Difference in refractive indices $n_{o}-n_{e}$ vs pressure in $\mathrm{GaS}$.
This point, plus the decrease in the index anisotropy under pressure, should not be taken as evidence for GaS evolving to a three-dimensional bonding scheme, where the difference in nature of interlayer van der Waals bonds and intralayer covalent-ionic ones would have disappeared as they became similar in strength. A counterexample which supports this statement may be found in hexagonal II-VII compounds where the bonding scheme is three-dimensional (ionic-covalent bonds are similar along the hexagonal axis and perpendicular to it) and where the index anisotropy may be positive, negative, or zero. The contributions to the dielectric function tensor may well cancel in such a way that a uniaxial crystal will have no index anisotropy at a given wavelength, and still retain an anisotropic bonding scheme. We propose that this is indeed the case for $\mathrm{GaS}$ under pressure.

\section{Elastic constants}

In a backscattering geometry, the Brillouin frequency shift is related to the velocity of sound, $V$, by

$$
\Delta v=\frac{2}{\lambda} \frac{n V}{c}
$$

where $c$ is the speed of light in vacuo, $\lambda$ the wavelength of the light, and $n$ the relevant refractive index. Sound velocities are related to the elastic constants by

$$
C_{i j}=\rho V^{2},
$$

where $C_{i j}$ is the appropriate combination of elastic constants for the particular propagation direction. $V_{1}(P), V_{\| \mid}(P), C_{11}(P)$, and $C_{33}(P)$ can thus be calculated since $n(P)$ and $\rho(P)$ have been determined as explained in the previous section. Our results are given in Fig. 7, which clearly shows a much stronger variation under pressure of interlayer parameters $\left(V_{\|}, C_{33}\right)$ than of intralayer ones $\left(V_{\perp}, C_{11}\right)$, as expected for a layer compound. In the range 0 to $17 \mathrm{GPa}, C_{11}$ which is connected to in-plane interactions varies by a factor 1.9 whereas $C_{33}$ which is related to interlayer compression varies by a factor of 4.5. This behavior of compression-restoring forces along $c$ is to be ascribed for the larger part to variations in the interlayer springs. It is completely analogous to the evolution of interlayer shear forces which has been previously studied by Raman scattering under high pressure of a shear rigid layer mode. ${ }^{11,20}$ Since the corresponding interlayer compression mode is $\mathbf{R a -}$ 


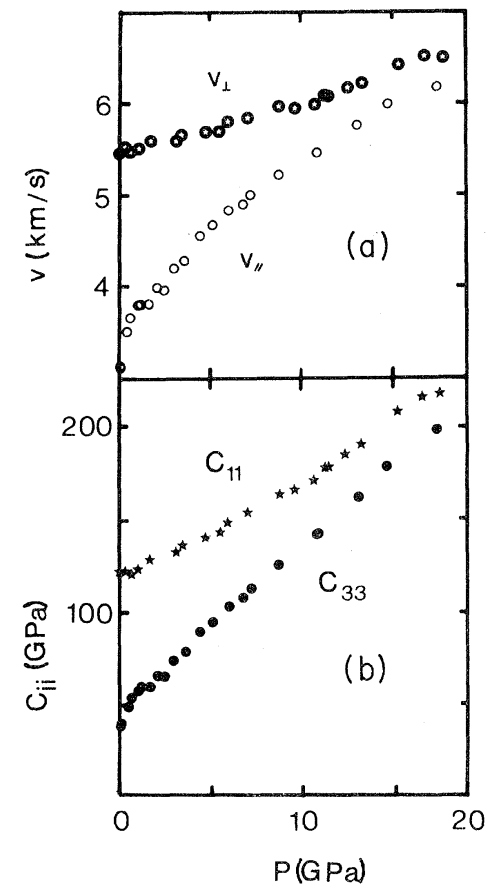

FIG. 7. (a) Longitudinal sound velocities in $\mathrm{GaS}$ along $c\left(V_{\|}\right)$and perpendicular to it $\left(V_{\perp}\right)$ vs pressure. (b) Corresponding variation of $C_{11}$ and $C_{33}$.

man inactive, the Brillouin experiment completes the picture of the behavior of the elastic forces in GaS: Under high pressure both shear and compression-restoring forces between the layers have the same magnitude as corresponding intralayer forces. However, as mentioned earlier, this does not imply that the nature of intra- and interlayer bonds has become identical; for instance, that interlayer bonding has become as covalent as intralayer bonding.

\section{Equation of state for $\mathrm{GaS}$}

In this section, we use the Murnaghan's equation of state ${ }^{21}$ to interpret the observed behavior of $\mathrm{GaS}$ under pressure. We choose it over Tait's $\mathrm{s}^{22}$ because of its simplicity and adaptability to an anisotropic case.

Murnaghan's equation of state (MEOS) is

$$
\rho=\rho_{0}\left(1+\frac{B_{0}^{\prime}}{B_{0}}\left(P-P_{0}\right)\right)^{1 / B_{0}^{\prime}},
$$

where $\rho, \rho_{0}$ are the densities at pressures $P$ and $P_{0}$, $B_{0}$ the bulk modulus at ambient pressure, and $B_{0}^{\prime}$ its derivative with respect to pressure.

As follows from Eq. (7), the bulk modulus is related to the density by

$$
B=B_{0}\left(\frac{\rho}{\rho_{0}}\right)^{B_{0}^{\prime}} .
$$

We have modified MEOS in the following manner in order to describe the behavior of anisotropic GaS.

(i) The bulk modulus for an elastically isotropic medium is

$$
B=C_{11}-\frac{4}{3} C_{44} \text {. }
$$

Assuming that the elastic constants have the same density dependence or $C_{44}<<C_{11}$, we may write Eq. (8) as

$$
C_{11}(P) \simeq C_{11}(0)\left(\frac{\rho}{\rho_{0}}\right)^{B_{0}^{\prime}} .
$$

(ii) The structural anisotropy of GaS is introduced by separating the contributions to $C_{11}$ and $C_{33}$ in the real crystal along the $a$ and $c$ directions as

$$
\begin{aligned}
& C_{11}=C_{11}(0)\left(\frac{a_{0}}{a}\right)^{3 B_{\perp}^{\prime}}, \\
& C_{33}=C_{33}(0)\left(\frac{c_{0}}{c}\right)^{3 B_{\|}^{\prime}} .
\end{aligned}
$$

Here, the derivatives $B_{\perp}^{\prime}$ and $B_{\|}^{\prime}$ are defined to coincide with $B_{0}^{\prime}$ in the isotropic case. In Fig. 8 we have plotted the logarithms of $C_{11}$ and $C_{33}$ versus $a_{0} / a$ and $c_{0} / c$, respectively, and indeed observe a linear relationship as predicted by Eq. (11). This does not entitle us to assume Eq. (11) as the correct EOS, but the relationship shows it to be an acceptable representation of the observed behavior. The slopes of the two curves in Fig. 8 yield the following values:

$$
B_{\perp}^{\prime}=2.2, \quad B_{\|}^{\prime}=5.3 .
$$

If we now compare with a three-dimensional compound built from atoms not too far away from $\mathrm{Ga}$ and $\mathrm{S}$ in the Periodic Table, we find that $\boldsymbol{B}_{0}^{\prime}$ is 4.7 for GaAs, ${ }^{23}$ that is much closer to $B_{\|}^{\prime}$ than to $B_{\perp}^{\prime}$. This is quite consistent with the fact that in low-dimensionality networks (molecular crystals, layer structures) external forces affect the weak intermolecular forces more than the intramolecular ones. ${ }^{20}$ That the intermolecular (interlayer) volume takes up the bulk of the compression is shown in the pressure variation of inter- and intramolecular (-layer) modes, i.e., the Grüneisen "constants" of the former are much closer to the values found in 


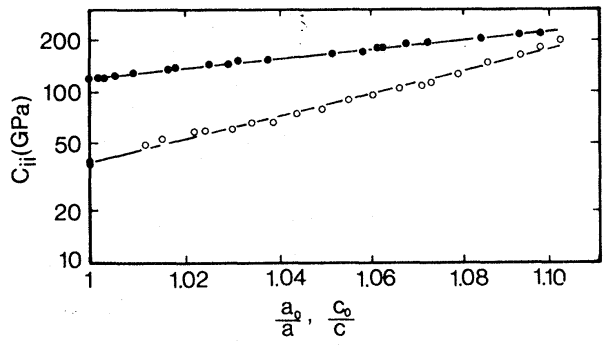

FIG. 8. Full circles: $C_{11}$ (log scale) vs $a_{0} / a$. Empty circles: $C_{33}$ (log scale) vs $c_{0} / c$. Straight lines depict the expected behavior for constant $B_{\perp}^{\prime}$ and $B_{\|}^{\prime}$ [Eq. (11)].

three-dimensional networks than those of the latter. ${ }^{11,20}$ The similarity of $B_{\|}^{\prime}$ which is dominated by the pressure behavior of the interlayer forces with a three-dimensional $B_{0}^{\prime}$ points to a similar conclusion. This is another example of the general trend in low-dimensionality networks: External properties (e.g. interlayer or intermolecular forces) have a three-dimensional character under pressure, whereas internal ones (e.g. intralayer, intramolecular) are singular.

\section{NOTE ON THE METHANOL-ETHANOL MIXTURE}

As noted before, we could follow the scattering from the 4:1 methanol-ethanol pressure-transmitting medium under pressure. The Brillouin shift at ambient pressure $\left(\Delta v \simeq 0.15 \mathrm{~cm}^{-1}\right)$ is much smaller than in GaS. The effect of pressure is shown in Fig. 9. To compute the velocity of sound, and $C_{11}$, we adopted the following procedure.

(i) Choice of EOS: Density data exist for methanol up to $5 \mathrm{GPa}^{24}$ Since the densities for both alcohols are similar within $2 \%$ up to $2 \mathrm{GPa}$, we take the density of the mixture to be given by the extrapolation of methanol density. Four EOS's were tested, namely, Tait's, Murnaghan's, Birch's and linear secant modulus. ${ }^{25}$ Only the former two gave a fit to experimental data and were retained. Tait's EOS (TEOS) ${ }^{23}$ is

$\rho=\rho_{0}\left[1-\frac{1}{1+B_{0}^{\prime}} \ln \left[1+\frac{1+B_{0}^{\prime}}{B_{0}}\left(P-P_{0}\right)\right]\right]^{-1}$.

It yields $B_{0}=0.7 \mathrm{GPa}$ and $B_{0}^{\prime}=10.5$, while Murnaghan's EOS (MEOS) [Eq. (7)] gives $B_{0}=1.2$ $\mathrm{GPa}$ and $B_{0}^{\prime}=7$. The extrapolation of these fits to

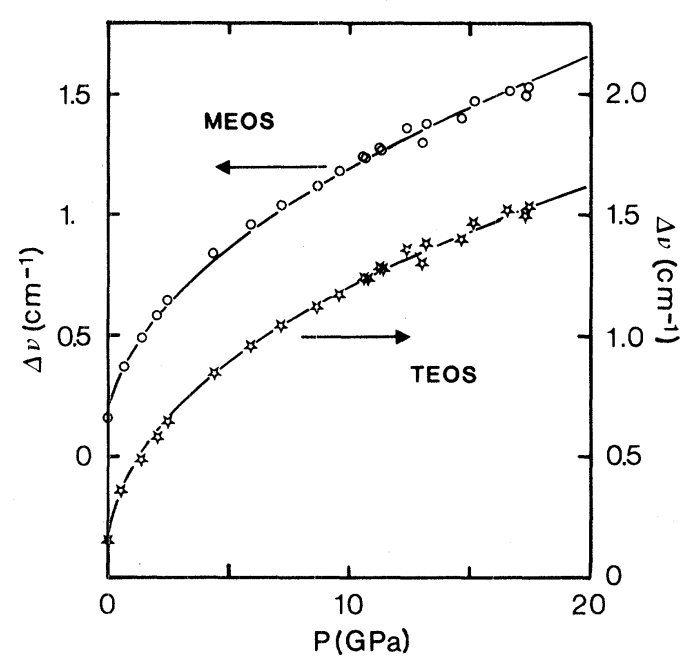

FIG. 9. Methanol-ethanol mixture. Experimental Brillouin shift: stars and empty circles are identical (right-hand and left-hand ordinates, respectively) and shifted by $0.5 \mathrm{~cm}^{-1}$ for clarity. Full lines are Murnaghan's EOS (upper line - left-hand scale) and Tait's EOS (lower line - right-hand scale). See text for discussion of equations of state.

18 GPa differ by $\sim 5 \%$.

(ii) The changes in refractive index under pressure have been shown ${ }^{26}$ to be very well described by a function of Eulerean strain $\epsilon$ for a number of liquids including methanol. From a fit to the results of Ref. 26 and recalling that $\epsilon$ is given by

$$
\epsilon=\frac{1}{2}\left[1-\left(\frac{\rho}{\rho_{0}}\right)^{2 / 3}\right],
$$

we find to a very good approximation

$$
n(\rho)=n\left(\rho_{0}\right)-0.571\left[1-\left(\frac{\rho}{\rho_{0}}\right)^{2 / 3}\right] .
$$

It is true that in evaluating $n(P)$ necessary for our calculation we must use $\rho(P)$ from one of the above-mentioned fits to the density. However, the changes in $n(P)$ depending on the choice of $\rho(P)$ are small and play no significant role in the interpretation of $V$ or $C_{i j}$.

We see from the previous paragraphs that the evaluation of $C_{11}$ will depend on the extrapolation chosen for $\rho(P)$. Figure 10 presents the values of $C_{11}$ calculated for both choices of the extrapolation. To obtain the fits shown in Fig. 10 we express the bulk modulus as a function of pressure by

$$
B(P)=B_{0}+B_{0}^{\prime}\left(P-P_{0}\right)
$$

for MEOS and by 


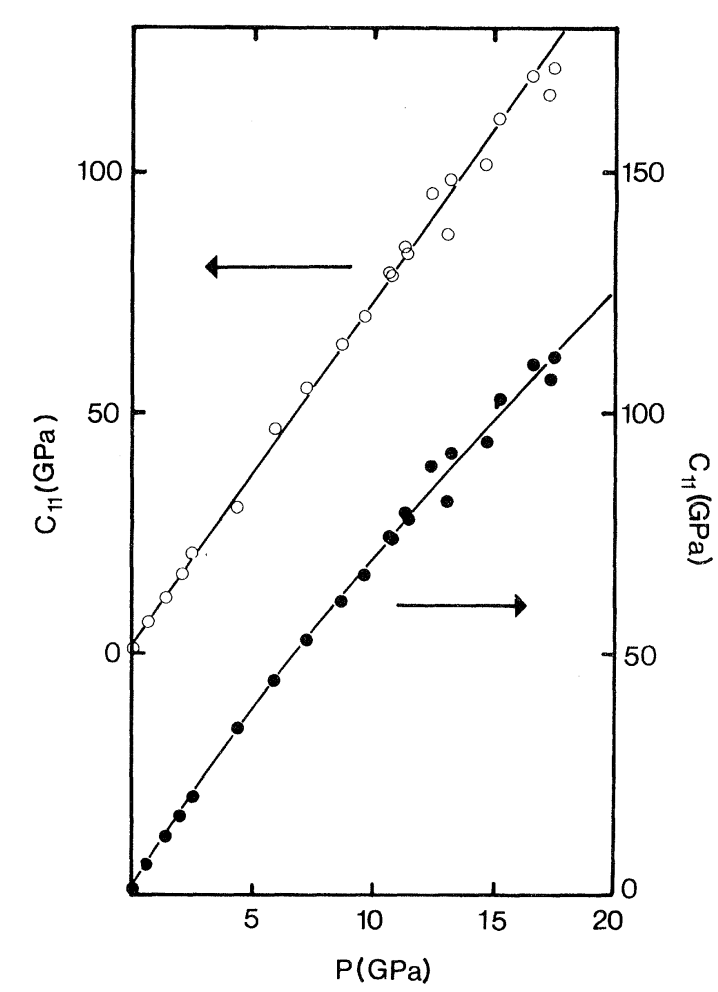

FIG. 10. $C_{11}$ for the ethanol-methanol mixture vs pressure. Circles are experimental values computed from $\Delta v$ 's of Fig. 9, along Murnaghan's EOS (empty circles - left-hand scale - upper curve - Eqs. (15) and (7)) and Tait's EOS [full circles - right-hand scale - lower curve - Eqs. (12) and (16)]. Shift of ordinates is for clarity.

$$
\begin{aligned}
B= & {\left[B_{0}+\left(1+B_{0}^{\prime}\right)\left(P-P_{0}\right)\right] } \\
& \times\left[1-\frac{1}{1+B_{0}^{\prime}} \ln \left[1+\frac{1+B_{0}^{\prime}}{B_{0}}\left(P-P_{0}\right)\right]\right]
\end{aligned}
$$

\section{for TEOS.}

In these equations we now replace $B$ by $C_{11}$ as we did for GaS. In this case, however, neglect of the term $\frac{4}{3} C_{44}$ in Eq. (9) is not as easy to justify since we expect a strong pressure dependence of $C_{44}$ on going from liquid to glass, i.e., $C_{44}$ is equal to zero in the liquid and different from zero in the glass. Nevertheless, our fit of Eqs. (15) and (16) to the data in Fig. 10 up to $10 \mathrm{GPa}$ (the plastic region) also holds above this range, that is in the hard glass region, within experimental dispersion. From the fits with MEOS we obtain $B_{0}=2.0 \mathrm{GPa}$ and $B_{0}^{\prime}=7.1$ and with TEOS $B_{0}=0.8 \mathrm{GPa}$ and $B_{0}^{\prime}=11.0$. We point out here that the fits are very insensitive to changes in $B_{0}$ : The ambient pressure bulk modulus may be varied by as much as $1 \mathrm{GPa}$ with no significant influence upon the fit. However, the values of $B_{0}^{\prime}$ determined from $C_{11}(P)$ are in good agreement with their respective values obtained from $\rho(P)$.

In Ref. 27, we have shown that for the case of van der Waals forces described by a LennardJones potential we expect $B_{0}^{\prime}=7$ in contrast to the case of a typical covalently bonded solid in which $B_{0}^{\prime} \simeq 4.5$. We conclude, therefore, that in the pressure range studied, the intermolecular forces in the ethanol-methanol mixture remain essentially van der Waals in nature.

\section{SUMMARY AND CONCLUSIONS}

We have made Brillouin scattering measurements up to $20 \mathrm{GPa}$ using the diamond anvil cell and have brought out the following points regarding the elastic behavior of GaS.

(1) The compressibility anisotropy along the $c$ axis and perpendicular to it narrows with pressure and is reversed at the highest pressure.

(2) The refractive index anisotropy rapidly decreases and becomes zero within the experimental error, above $14 \mathrm{GPa}$.

(3) $C_{33}$, and consequently the longitudinal velocity along the $c$ axis increase rapidly and, at $18 \mathrm{GPa}$, become comparable to corresponding quantities in the layer plane.

(4) The Raman data on interlayer shear modes show that, under high pressure, interlayer bonds are as strong as - or stronger than - intralayer ones but the layer structure still prevails. Restoring forces on atoms become more isotropic with pressure since their magnitude within the layer and in the interlayer space become comparable, but they do retain their individual behavior under strain and follow each a different equation of state up to the highest pressures [Eq. (11) - Fig. 8]. This means that the bulk of the compression, which was taken at low pressures by the interlayer space, is taken (Fig. 2) at high pressure by the layers themselves.

(5) Simultaneous measurement of the refractive indices by means of interference fringes, and of compressibilities either by optical or by $x$-ray methods allows Brillouin scattering measurements to be extended effectively to the full range of the diamond anvil cell, that is several tens of GPa.

(6) Brillouin scattering methods will be a unique tool to obtain elastic constants under pressure on 
small monocrystalline samples. Further, it is possible to heat the sample by an appropriate laser power. This is important in geophysics or tectonophysics where physical quantities are needed not only under high pressure but also at high temperatures.

\section{ACKNOWLEDGMENTS}

The authors wish to thank M. Cardona for critical reading of the manuscript. This study has been supported in part through Contract DRET No. 80 34500004707501 .
*Present address: Argonne National Laboratory, 9700 South Cass Avenue, Argonne, Ill. 60439.

${ }^{1}$ F. F. Voronov, E. V. Chernycheva, and V. A. Goncharova, Fiz. Tverd. Tela (Leningrad) 21,100 (1979) [Sov. Phys. - Solid State, 21, 59 (1979)].

${ }^{2}$ F. F. Voronov, V. A. Goncharova, and O. V. Stalgorova, Zh. Eksp. Teor. Fiz. 76, 1351 (1979) [Sov. Phys._JETP 49, 687 (1979)].

3 J. Frankel, F. J. Rich, and C. G. Homan, J. Geophys. Res. 81, 6357 (1976).

4J. Frankel, M. A. Hussain, and R. D. Scanlon, J. Phys. Chem. Solids 40, 67 (1979).

${ }^{5}$ H. K. Mao and P. M. Bell, Science 200, 1145 (1975).

${ }^{6}$ G. J. Piermarini and S. Block, Rev. Sci. Instrum. $\underline{46}$, 973 (1975).

${ }^{7}$ H. K. Mao, P. M. Bell, J. W. Shaner, and D. J. Steinberg, J. Appl. Phys. 49, 3276 (1978).

8J. M. Besson and J. P. Pinceaux, Rev. Sci. Instrum. 50, 541 (1979).

${ }^{9}$ C. H. Whitfield, E. M. Brody, and W. A. Bassett, Rev. Sci. Instrum. 47, 942 (1976).

${ }^{10}$ H. Shimizu, E. M. Brody, H. K. Mao, and P. M. Bell, Phys. Rev. Lett. 47, 128 (1981).

${ }^{11}$ A. Polian, J. C. Chervin, and J. M. Besson, Phys. Rev. B 22, 3049 (1980).

12J. M. Besson, J. Cernogora, and R. Zallen, Phys. Rev. B 22, 3866 (1980).

${ }^{13}$ T. A. McMath and J. C. Irwin, Phys. Status Solidi A
38, 731 (1976).

${ }^{14}$ M. Mejatty, A. Segura, R. Letoullec, A. Chevy, and H. D. Fair, J. Phys. Chem. Solids 39, 25 (1978).

15J. F. Nye, Physical Properties of Crystals (Clarendon, Oxford, London, 1967).

${ }^{16}$ A. Kuhn, A. Chevy, and R. Chevalier, Acta Crystallogr. B 32, 983 (1976).

${ }^{17}$ B. M. Powell, S. Jandl, J. L. Brebner, and F. Levy, J. Phys. C $\underline{30}, 3039$ (1977).

${ }^{18}$ G. Hamaguchi, K. Wasa, and M. Yamawaki, Third International Conference on Phonon Scattering in Condensed Matter, Providence, 1979.

${ }^{19}$ A. Polian (unpublished).

${ }^{20}$ R. Zallen and M. L. Slade, Phys. Rev. B $\underline{18}, 5775$ (1978).

${ }^{21}$ F. D. Murnaghan, Proc. Nat. Acad. Sci. $\underline{30}, 244$ (1944).

22J. R. McDonald, Rev. Mod. Phys. 38, 669 (1966).

${ }^{23}$ M. J. McSkimin, A. Jayaraman, and P. Andreatch, J. Appl. Phys. 38, 2362 (1967).

24P. W. Bridgman, Proc. Acad. Sci. 499, 3 (1913); 74, 399 (1942)

${ }^{25} \mathrm{~A}$ review on the equations of states is given in: J. R. McDonald, Rev. Mod. Phys. 41, 316 (1969).

${ }^{26} \mathrm{~K}$. Vedam and P. Limsuwan, J. Chem. Phys. $\underline{69}, 4762$ (1978).

${ }^{27}$ A. Polian, J. M. Besson, M. Grimsditch, and H. Vogt, Appl. Phys. Lett. 묘, 334 (1981); 죠, 1033 (1981). 